\title{
EDITORIAL
}

\section{Challenges and Facilities in Health Sector in Bangladesh}

Bangladesh has made big strides in the Health Sector. Visibly there are various proliferations in health infrastructures such as medical colleges, medical university, private medical colleges, private clinics, private hospitals, district hospitals, rural health complexes (RHC) and community clinics. Much progress has been made in the pharmaceutical sector providing various kinds of medicines, I/V fluids, anticancer drugs etc. at affordable price.

\section{Basic statistics:}

Population density is 926 per sq $\mathrm{km}$ with crude birth rate 20.8 per 1000 and life expectancy 69/70 yrs. Bangladesh (BNHA estimates) per capita health expenditure is US $\$ 16$ (3.6\% of GDP).. Population per physician is 3012 . Population per bed is 2665. Population per nurse is 6342. Total registered graduate doctors stand 53063; percentage of doctors working under the private sector is $58 \%$ (2011).

We have 421 Upazila Health Complex (DGHS), 14,025 Community Clinics (3,975 CCs to be added soon) [DGHS \& DGFP], 30,000 Satellite Clinic (DGFP) and 36,764.

Community Nutrition Centers (NNP). Our aim is to reduce child mortality rate by $2 / 3$ and the maternal mortality rate by $3 / 4$ by the year 2015 .

\section{Health problems in Bangladesh:}

While rural problems are malnutrition, worm infestations, diarrhoea, anemia, poor housing, poor sanitation, Malaria, Kalazar, ARI etc, in urban areas most prevalent diseases are Hypertension (HTN), Diabetes mellitus (DM), Heart diseases, Cancers, Dengue, air pollution, sound pollution, Sexually transmitted diseases (STDs). Non communicable diseases have shoot up as a result of increased longivity, life style changes, industrialisation and work stress. Various types of cancer, coronary artery diseases, strokes, chronic renal diseases, chronic liver diseases, COPD has taken over mortality figures in contrast to infectious diseases. Newer outbreaks often happen like dengue, Chikangunya, Nippah, Anthrax, Bird flu. As per ICDDRB, Dhaka office (2009), among the diarrhea cases 22.2\% were due to vibrio cholera, $23.2 \%$ were rota vrus infection and 39\% had no pathogen. Only 26.3\% people wash their hands with soap while only $7 \%$ wash after defecation. Only 3\% people wash their hand with soap before having a meal or feeding children.

Over 50,000 tube wells have been analyzed with 63\% being found to be contaminated with arsenic at a concentrations above the acceptable limit of $0.05 \mathrm{mg} / \mathrm{l}$. Currently over 4,000 cases of Arsenicosis have been diagnosed and many more individuals are considered at risk.

AIDS prevalence has increased in the two southwestern districts in Satkhira and Jessore upto December, 2013. An estimated 3,241 Bangladeshis were found to have HIV/AIDS positive. This is less than 0.1 percent of its population. AIDS/HIV prevalence is 0.3 percent in India and 0.4 percent in Nepal.

One third of TB patients still out of reach: Although hundreds of thousands of lives have been saved due to the National Tuberculosis Control Programme (NTP), it is estimated that about one third of the tuberculosis patients have been out of reach of such programmes. While 1,84,492 people were diagnosed as having TB in 2013, the actual number of people who remain undetected and who may spread the disease is unknown. There is a resurgence of kalazar after 1990. Population are affected in 40 districts and 120 upazilas. Number of patients with kalazar is more than 20 thousand. According to WHO, every year 2,50,000 suffer from cancers and 1,50,000 die without adequate treatment. We have dirty environment, river pollution and drying up of river, inadequate work place safety (occupational hazards). One in eight dies for air pollution. The problems of rape, acid injuries, domestic violence, street violence, traffic accidents has gained increased magnitude known more so because of free media coverage. Crush injuries due to building collapse, burn cases due to political violence, drowning in the inland water transport sector, electrocution due to lightening, domestic electrical shocks and burns are becoming increasingly important health issues. Poisoning both at domestic and street levels contributes to unacceptable mortality and 
morbidity. Adulteration of food is an epidemic now. Toxicology still a new term in our health sector..Different types of poisonous snake are frequently found in our riverine, agricultural and bushy lands. Insects' sting and wasp bees are also common in our country.

Scientific disposal of hospital waste is of great concern; effluent from industries are polluting natural river. Drug addiction has become a major threat and challenges to the society particularly the young generation and the dejected slum dwellers. Climatic change is adversely affecting a large segment of population. Medical waste disposal, blood transfusion, fake care giver, fake medicine and appliances are continuing problems of our health sector.

\section{Achievement:}

A satisfactory level of progress has been made in family planning. The population growth rate has been brought down. We have a good antihelminth coverage (99\%) as we all know about the soil contamination of this country. We still need to do a lot to stop outbreak of water borne diseases. In Bangladesh, $74 \%$ of the population has access to safe drinking water. Tube well is rendering great contribution to water supply. Urban areas have ( $25 \%$ of the population) $68 \%$ tube wells and rural areas (75\% of the population) $96 \%$ tube wells. $13.5 \%$ of rural households use sanitary latrines. Overall access including simple pit latrine is $43.4 \%$ with an increase of about $1 \%$ per annum. Specific Day is observed in Bangladesh as elsewhere in the world to raise awareness about the TB patients who are "missed" by anti-TB programmes. There has been significant gain in terms of Polio \& Small Pox eradication. Extensive vaccination, case isolation has resulted in diminishing number of Diphtheria and Tetanus. Country has been delared as polio free. A great number of tuberculosis satellite clinics now offer free treatment with contribution from NGOs. $\mathrm{HIV} / \mathrm{AIDS}$ is at a low level of prevalence but remains a threat because of international employment. Kalazar and Malaria have been significantly curbed down. Due to anti-HIV/AIDS awareness, there is a beginning to reverse the spread of HIV/AIDS. The incidence of TB is also decreasing.

In 2008, there were 84,000 malaria patients which had gone down to 26,000 by 2013. Deaths due to malaria were 154 in 2008 while it came down to 74 in 2103 . Our target is to have 0 death for malaria in 2020. In 2013 number of Kalazar dropped down to 1500 and our target is to eliminate Kalazar by 2015.

\section{Conclusion:}

We have the infrastructure. We need to address the whole spectrum of issue, challenges, and threats that are required to be systematically analysed and pragmatically solved. At the present time, though 35 public and 63 private medical colleges offers 8727 seats, quality education and appropriate training remain a major concern. There are occasions in hospital and clinics where management failures often lead to strikes, violence and damage to institutions. Bangladesh has a dynamic and vibrant society. There is more to achieve.

\section{Acknowledgement:}

"Challenges and facilities in health sector in Bangladesh" has been extrapolated from "Prof. MAQUADERI MEMORIAL ORATION" at $25^{\text {th }}$ Annual scientific conference of the Association of Physicians of Bangladesh (APB) delivered by Prof. (Major General) Rabiul Hossain, Consultant Physician General, Bangladesh Armed Forces.

\section{Poisoning and Toxicology}

Poisoning is a major cause of morbidity and mortality in Bangladesh. It remains among the top ten health problems in Bangladesh according to the health report of Bangladesh health authority.

Among different agents of poisoning, pesticides remain the most important one in terms of incidence and severity in Bangladesh.
In Bangladesh, there was a changing pattern of poisoning during last two decades; homicidal poisoning like commuters poisoning are increasing every year and recently in tertiary care centres, it has become number one poisoning problem. The unspecified poisoning is also a leading problem in tertiary centres. As toxicological confirmation is lacking and management 
is also difficult, toxidrome could not be applied due to nonspecific presentation.

Clusters of different poisoning is also rising in Bangladesh at different location like copper and Puffer fish in southern area, and aluminium phosphate poisoning in eastern area. Herbal products, methanol, hydrocarbon, household products specially corrosive ones are also commonly consumed poisons. Suicidal copper sulphate poisoning is prevalent in southern parts of Bangladesh. It has high mortality rate, death occurs due to acute renal failure and acute hepatic failure

Poisoning was the second of top ten diseases dealt in medicine wards of Dhaka medical college hospital in 2012. Acute poisoning accounts for 1\% of hospital admission in the UK. Even in developed worlds poisoning is a major cause of death in young adults, most death occurred before patients reach medical attention. The mortality is much lower than $1 \%$ in those admitted to hospital. Most important thing is that much of these deaths are preventable if appropriate measures are taken at right time.

Besides organophosphorus and commuter poisoning, poisonous snake envenoming is a reality in our riverine and agricultural land. Varieties of Kraits, Cobras, Green viper are identified as notorious snakes living widespread in the bushy land of ours. Many of us are not acquainted with the syndromic effect of their poisons. We are yet to develop ways to diagnosis the species and the ways to evaluate the effect of antisnake venom. Anti- snake venom is not being manufactured in our country; we are to depend on the poly valent antivenom from the neighbouring country.
We need to come out of the traditional belief and introduce state-of-the art management of envenoming.

There was an outbreak of intoxication with Puffer fish tetradotoxin where 91 consumed the fish with 14 casualties in costal belt in 2008 . There was another report of Ghagra shak intoxication in 2007 in Sylhet where 81 persons identified and 4 died with severe liver damage.

The medically important groups of (insects) Hymenoptera are the Apoidea (bees), Vespoidea (wasps, hornets, and yellow jackets), and Formicidae (ants) who deliver their venom by stinging their victims. Hymenopterid stings and subsequent allergic reactions are a common indication for emergency department visits worldwide including Bangladesh. Unrecognized anaphylactic reactions to hymenopterid stings is a significant cause of sudden and unanticipated deaths among young people, with or without atopic histories. Unfortunately, severe cases of insect bites are still underreported in our country and no taxonomical study so far been done in Bangladesh. Deliberate contamination of food materials with prohibited, inferior or deleterious substances is called adulteration. Adulteration has reached an epidemic proportion recently. All types of agricultural, dairy, poultry, fishery, packaged, bottled and canned food are adulterated. The whole population, sadly, has been slow poisoned.

We need to adopt public health policy like different communicable diseases for mass awareness regarding the wide and far-reaching adverse and detrimental effects of poisons and toxins. 